\title{
Modeling a Negative Response Bias in the Human Amygdala by Noradrenergic-Glucocorticoid Interactions
}

\author{
Juraj Kukolja, ${ }^{1,2,4}$ Thomas E. Schläpfer, ${ }^{2}$ Christian Keysers, ${ }^{5}$ Dietrich Klingmüller, ${ }^{3}$ Wolfgang Maier, ${ }^{2}$ Gereon R. Fink, \\ René Hurlemann ${ }^{2}$ \\ ${ }^{1}$ Cognitive Neurology Section, Institute of Neuroscience and Biophysics-Medicine, Research Centre Juelich, 52425 Juelich, Germany, Departments of \\ ${ }^{2}$ Psychiatry, and ${ }^{3}$ Clinical Biochemistry, Division of Endocrinology, University of Bonn, 53105 Bonn, Germany, ${ }^{4}$ Department of Neurology, University \\ Hospital, Cologne University, 50924 Cologne, Germany, and ${ }^{5}$ Behavioral and Cognitive Neuroscience NeuroImaging Center, University Medical Center \\ Groningen, 9713 AW Groningen, The Netherlands
}

An emerging theme in the neuroscience of emotion is the question of how acute stress shapes, and distorts, social-emotional behavior. The prevailing neurocircuitry models of social-emotional behavior emphasize the central role of the amygdala. Acute stress leads to increased central levels of norepinephrine (NE) and cortisol (CORT), and evidence suggests that these endogenous neuromodulators synergistically influence amygdala responses to social-emotional stimuli. We therefore hypothesized that amygdala responses to emotional facial expressions would be susceptible to pharmacologically induced increases in central NE and CORT levels. To specifically test this hypothesis, we measured amygdala activation to emotional faces using functional magnetic resonance imaging in 62 healthy subjects under four pharmacological conditions: (1) single oral dose of placebo, (2) $4 \mathrm{mg}$ of the selective NE-reuptake inhibitor reboxetine (RBX), (3) $30 \mathrm{mg}$ of hydrocortisone, or (4) both drugs in combination. We found that a decrease in amygdala activation to positive facial emotion was coupled with an increase in amygdala activation to negative facial emotion in the RBX-CORT combined challenge condition. In conclusion, a pharmacologically induced elevation of central NE and CORT levels in healthy subjects created a negative response bias in the amygdala that did not exist at baseline. Our results implicate a causative role of NE-CORT interactions in the emergence of a negative bias of cognitive and emotional functions which is germane in stress-related affective spectrum disorders.

Key words: norepinephrine; cortisol; amygdala; emotion; stress; major depressive illness

\section{Introduction}

Rapid and flexible adaptation to acute stress is of key importance across species to maximize chances of survival; however, when chronic, disproportional in intensity, or unrelated to genuine threats, stress may become maladaptive and precipitate severe affective spectrum disorders including anxiety disorders (AXD) and major depressive disorder (MDD) (Gold et al., 1988; Wong et al., 2000; Southwick et al., 2005).

A prominent dysfunction in these disorders is a negative cognitive bias in the patients' appraisal of people and life events (Beck, 1967; Chamberlain and Sahakian, 2006). In experimental settings, a negative cognitive bias becomes evident when MDD

\footnotetext{
Received July 30, 2008; revised 0ct. 14, 2008; accepted 0ct. 14, 2008.

This work was supported by a German Federal Ministry of Education and Research (BMBF) Grant (01GW0671) (to R.H.), a German Research Foundation (DFG) Grant (HU1302/2-1) (to R.H.), a Netherlands Organization for Scientific Research (NWO) Vidi Grant (to C.K.), and a European Commission Marie Curie Excellence Grant (to C.K.). We are grateful to C. Frahnert, H. Kölsch, and P. Wegener for analyzing blood samples and acknowledge the invaluable help of the Magnetic Resonance and Cognitive Neurology groups located at the Institute of Neuroscience and Biophysics-Medicine.

The authors declare no conflict of interest.

Correspondence should be addressed to either of the following: Dr. Juraj Kukolja, Department of Neurology, University Hospital, Cologne University, Kerpener Strasse 62, 50924 Cologne, Germany, E-mail: juraj.kukolja@ukkoeln.de; or Dr. René Hurlemann, Department of Psychiatry, University of Bonn, Sigmund-Freud-Strasse 25, 53105 Bonn, Germany, E-mail: renehurlemann@me.com.

D0I:10.1523/JNEUROSCI.3592-08.2008

Copyright $\odot 2008$ Society for Neuroscience $\quad$ 0270-6474/08/2812868-09\$15.00/0
}

patients exhibit more intense psychophysiological responses to emotionally negative stimuli and less intense responses to emotionally positive stimuli (Chamberlain and Sahakian, 2006). Paralleling these behavioral observations, neuroimaging studies have demonstrated a hyperresponsiveness (i.e., relative to healthy controls) of the amygdala toward emotionally negative stimuli in patients with AXD (Stein et al., 2002; Phan et al., 2006; Etkin and Wager, 2007; Stein et al., 2007) and MDD (Siegle et al., 2002; Dannlowski et al., 2007). Despite enormous implications for an advanced understanding and pharmacological treatment of these disorders, little is known about the neurochemical underpinnings of a negative response bias in the amygdala.

One intriguing possibility is that stress modulates amygdala function in a way that could predispose an individual to AXD and MDD. Indeed, dysregulations of two major endogenous neuromodulators linked to stress, i.e., norepinephrine (NE) and cortisol (CORT), have been documented in AXD patients (Abelson and Curtis, 1996; van West et al., 2008) as well as in MDD patients (Burke et al., 2005). Consequently, current etiological concepts of AXD and MDD firmly emphasize the crucial role of hypercortisolism (de Kloet, 2003; Young et al., 2004; Burke et al., 2005) and noradrenergic overdrive (Wong et al., 2000; Charney, 2004). The major source of NE release in the amygdala is the locus ceruleus (LC) located in the dorsorostral pons (Asan, 1998; Berridge and Waterhouse, 2003). LC-NE signaling is critically involved in the 
primary stress reaction mediating a rapid fight-or-flight response. In a second, slower step of the stress response, the hypothalamo-pituitary-adrenal (HPA) axis is activated, leading to increased peripheral and central levels of CORT (Herman et al., 2005). Importantly, pharmacological challenge studies in rodents and humans suggest that the amygdala is a critical locus of NE and CORT interactions (Quirarte et al., 1997; Roozendaal et al., 2006b; van Stegeren et al., 2007). Converging evidence from these studies suggests that the modulatory effects of CORT on amygdala function require the presence of NE.

We therefore hypothesized that elevated levels of NE and CORT together mediate a response bias in the amygdala, which facilitates processing of negative stimuli at the cost of positive stimuli and thus potentially contributes to the onset and maintenance of stress-related affective spectrum disorders including AXD and MDD.

To specifically test this hypothesis, we studied 62 healthy subjects in a double-blind, randomized, placebo-controlled design using functional magnetic resonance imaging (fMRI). Subjects were exposed to a facial emotion paradigm after administration of single oral doses of either placebo, the selective NE-reuptake inhibitor reboxetine (RBX) (4 mg), hydrocortisone (synthetic CORT) $(30 \mathrm{mg}$ ) or both drugs in combination. The rationale was to determine whether pharmacologically elevated levels of NE, CORT, or both in combination increase amygdala responses to fearful faces and/or decrease amygdala responses to happy faces. Additionally, we studied potential pharmacological effects on postscan emotion ratings and their relationship to the fMRI data.

\section{Materials and Methods}

Subjects. Sixty-two healthy young subjects (students at the University of Aachen and trainees at the Research Center Juelich, recruited by local advertisement), participated in the present study (for sample characteristics, see Table 1). All subjects were right-handed, as assessed by the Edinburgh Handedness Inventory (Oldfield, 1971) and had normal or corrected-to-normal vision. Informed written consent was given by all subjects before participation. The study had full ethical approval. Before participation, subjects completed a medical questionnaire to exclude psychiatric or neurologic disorders, pregnancy or breast feeding, intake of any medication interfering with either RBX or CORT, allergic reactions to RBX or CORT, infectious diseases, heart diseases, arterial hypertension, inflammatory bowel disease, osteoporosis, liver or kidney dysfunction, diabetes mellitus, psoriasis, hormonal diseases (such as Cushing's disease), glaucoma, urinary retention, or prostatic hyperplasia. Additionally, heart rate, blood pressure, and an electrocardiogram were obtained. Subjects underwent a brief neuropsychological and psychiatric assessment including a measure of verbal IQ based on lexical decisions [MWTB (Mehrfachwahl-Wortschatz-Test)] (Lehrl, 1995), the BDI (Beck Depression Inventory) (Beck et al., 1995), and the M.I.N.I. (Mini International Neuropsychiatric Interview) (Lecrubier et al., 1997). In brief, subjects had no neuropsychological impairments, nor did they have any psychopathology. One subject was excluded from further analysis due to excessive head movements during the fMRI session.

Drug administration and analysis. In a between-subjects, double-blind study design, participants were randomly assigned to one of four groups, the first of which ("Plac") received four tablets of saccharose placebo in a sealed cup, the second ("Cort-only") received three tablets of hydrocortisone $(10 \mathrm{mg}$ each) and one tablet with saccharose placebo, the third ("Rbx-only") received one tablet of reboxetine mesilate $(4 \mathrm{mg}$ ) and three tablets of saccharose placebo, and the last ("Rbx-Cort") received one tablet of reboxetine mesilate $(4 \mathrm{mg})$ and three tablets of hydrocortisone (10 mg each). RBX is a highly selective inhibitor of presynaptic NE reuptake, thus increasing the availability of NE in the synaptic cleft (Kent, 2000; Scates and Doraiswamy, 2000). A $4 \mathrm{mg}$ single oral dose of RBX was administered in analogy to previous studies investigating noradrenergic effects on emotion and cognition (Papps et al., 2002; Hurlemann et al.,
2005; Norbury et al., 2008). A $30 \mathrm{mg}$ single oral dose of hydrocortisone was administered to elevate CORT activity to levels ranging between moderate $(20 \mathrm{mg})$ and extreme $(40 \mathrm{mg})$ acute stress (Abercrombie et al., 2003).

Drugs were given $1 \mathrm{~h} 45 \mathrm{~min}$ before scanning. This interval was chosen because (1) maximum RBX serum concentrations in humans are reached $2 \mathrm{~h}$ after oral intake (serum half-life $13 \mathrm{~h}$ ) (Fleishaker, 2000) and because (2) modulatory effects of CORT on amygdala neuronal activity can be expected 1-2 h after oral intake (Czock et al., 2005). These effects of CORT appear to be mediated by genomic actions via intracellular glucoand mineralocorticoid receptors (Joëls, 2006).

Heart rate and blood pressure were measured once before drug administration and once before scanning. Furthermore, subjects completed self-rating scales assessing subjective discomfort due to physical complaints [B-L (Beschwerdeliste)] (von Zerssen and Koeller, 1976b) and subjective mood [Bf-S (Befindlichkeitsskala)] (von Zerssen and Koeller, 1976a) before drug administration as well as before and after scanning.

For an assessment of CORT levels, saliva samples were collected immediately before drug administration as well as before and after scanning from each subject using Salivette collection devices (Sarstedt) and stored at $-20^{\circ} \mathrm{C}$ until biochemical analysis. Free salivary CORT levels were measured using a commercially available radioimmunoassay (CORTCT2, CIS Bio International). After scanning, a blood sample was collected for the determination of RBX levels and total CORT levels (for cross-validation of the salivary values). Plasma concentrations of RBX were analyzed by high-performance liquid chromatography (HPLC). The chromatographic separation was achieved on a Nucleosil 100Protect 1 column (MACHEREY-NAGEL and CoKG) with acetonitrilepotassium dihydrogenphosphate buffer as mobile phase. The CORT plasma level analyses were performed by an extramural biomedical laboratory (Gaal, Bonn, Germany) using a fluorescence polarization immunoassay (FPIA) technique.

$f M R I$ paradigm. The fMRI stimulation paradigm consisted of a pseudorandom series of film clips obtained from 10 professional actors (five females, five males) who in each these clips displayed either a happy, fearful, or neutral facial expression in a standardized manner (van der Gaag et al., 2007) (see supplemental material, available at www. jneurosci.org). In the emotionally neutral movies, actors blew up their cheeks, which served to control for facial movements in the emotionally salient movies. Note that in previous fMRI studies, no differences were found between amygdala responses to fearful, happy, or neutral movies (van der Gaag et al., 2007), making these stimuli an ideal imaging probe to study pharmacogenic response bias in the amygdala. Furthermore, we used dynamic instead of static stimuli for the reason of higher ecological validity: in everyday life, during social interactions, dynamic facial expressions rather than static facial displays serve as the primary conveyors of social-emotional information (see also Hurlemann et al., 2008). We thereby adapted an approach used in recent single-neuron recording studies of the primate amygdala (Kuraoka and Nakamura, 2007). Each movie had a duration of three sec and was presented three times, resulting in 30 stimulus presentations per condition. Movies occurred at a rate of one every $10.8 \mathrm{~s}(6.3-15.3 \mathrm{~s})$ over a period of $21.2 \mathrm{~min}$. The same facial expression never occurred in immediate succession. A fixation cross was interspersed between each film clip. During fMRI scanning, subjects were engaged in an emotion discrimination task requiring appropriate push-button responses. Stimulus delivery and response recording was performed using Presentation11 (Neurobehavioral Systems). A mirror system was used for stimulus presentation (viewing distance, $254 \mathrm{~cm}$ ). Stimuli subtended a visual angle of $8.2^{\circ}$ horizontally and $6.5^{\circ}$ vertically. After scanning, subjects were asked to watch all film clips again on a laptop screen and to rate them with respect to arousal and pleasure on a scale ranging from 1 to 9 . Ratings were analyzed using repeated-measures ANOVAs, with the between-subject factors RBX (RBX, no RBX), CORT (CORT, no CORT) and the within-subject factor emotional facial expression (henceforth called "emotion," with the levels fearful, happy, and neutral). Significant results of post hoc tests are reported after Bonferroni-correction for multiple comparisons.

fMRI hardware and procedures. Functional MRI images were acquired 
using a TRIO $3 \mathrm{~T}$ whole-body scanner (Siemens) equipped with a standard head coil for radiofrequency transmission and signal reception. Sequence parameters were: $\mathrm{T} 2^{\star}$-weighted echoplanar images (EPI) with bloodoxygenation level-dependent (BOLD) contrast, echo time $(\mathrm{TE})=31 \mathrm{~ms}$, repetition time $(\mathrm{TR})=2000 \mathrm{~ms}$, flip angle $=90^{\circ}$, slice thickness $2.0 \mathrm{~mm}$, interslice gap $1.0 \mathrm{~mm}$, field of view $(\mathrm{FoV})=200 \mathrm{~mm}$, matrix size $88 \times 88$, in-plane resolution $=2.273 \mathrm{~mm} \times 2.273 \mathrm{~mm}$. Twentyeight axial slices per volume were positioned at an angle between a line crossing the anterior and posterior commissure (AC-PC line) and a line paralleling the medial tentorium cerebelli and thus approximated slice orientations which were proposed to reduce susceptibility artifacts in the amygdala (Deichmann et al., 2003; Weiskopf et al., 2006). In addition, a high-resolution $\mathrm{T} 1$ anatomical image was obtained from each subject using a standard 3D magnetizationprepared, rapid acquisition gradient echo sequence. During scanning, heart rate was monitored via pulse oxymetry. The fMRI time series consisted of 641 images. The first five images during which instruction trials were shown were discarded from further analysis.

Image processing. The image preprocessing was performed using Matlab7 (The MathWorks) and SPM5 (http://www.fil.ion.ucl.ac.uk/spm). After realignment the EPI images were spatially normalized to the MNI single subject template (Montreal Neurological Institute) provided by SPM5 and resampled at $2 \times 2 \times 2 \mathrm{~mm}^{3}$ voxel size. The normalized images were spatially smoothed using a $4 \mathrm{~mm}$ full-width-half-maximum Gaussian kernel to meet the statistical requirements of the General Linear Model and to compensate for residual anatomical variations across subjects.

The data were analyzed using a general linear model as implemented in SPM5 (Kiebel and Holmes, 2003) in an event-related manner. Each experimental condition was modeled using a boxcar reference vector convolved with a canonical hemodynamic response function and its firstorder temporal derivative. Low-frequency signal drifts were filtered using a cutoff period of $128 \mathrm{~s}$. Parameter estimates were subsequently calculated for each voxel using weighted least squares to provide maximum likelihood estimators (Kiebel and Holmes, 2003). No global scaling was applied.

For each subject, simple main effects for each condition were computed using baseline contrasts. These first-level individual contrasts were then entered into a second-level group analysis using an ANOVA with the between-subject factors RBX (RBX, no RBX), CORT (CORT, no CORT), and the within-subject factor emotion (happy, neutral, fearful) using a random effects model (Penny and Holmes, 2003). Violations of sphericity were accounted for by modeling nonindependence across parameter estimates from the same subject and allowing unequal variances both between conditions and subjects.

For a hypothesis-driven analysis of the amygdala, the left and the right amygdalae were defined as regions of interest (ROIs) based on cytoarchitectonic maximum probability maps (MPM) (Amunts et al., 2005) using the SPM Anatomy toolbox (Version 1.4, http://www.fz-juelich.de/ime/ spm_anatomy_

toolbox (Eickhoff et al., 2005)). The cytoarchitectonic maximum probability maps used for ROI definition denote the most likely anatomical area at each voxel of the MNI single subject template based on probabilistic cytoarchitectonic maps derived from a sample of 10 human postmortem brains, which were subsequently normalized to the MNI space (Amunts et al., 2005; Eickhoff et al., 2006). The amygdaloid ROI used in the present study covered the centromedial, superficial, and laterobasal groups of amygdaloid nuclei (Amunts et al., 2005).

To identify regions differentially active as a function of emotion and pharmacological condition, we used F-contrasts to test for the main ef- fect of emotion (happy, neutral, fearful); the main effect of RBX; the main effect of CORT; the two-way interaction effects between RBX and emotion and between CORT and emotion; and the three-way interaction between RBX, CORT, and emotion. Activations yielded by the resulting SPM(F) maps are reported at $p<0.05$, corrected for family-wise error (FWE) by reference to the Gaussian random field theory (Worsley et al., 1996).

To disentangle these effects, we performed subsequent tests for simpler interactions or simple main effects, whenever applicable, within the clusters found active in the primary analysis. For these post hoc tests, FWE correction was applied only for the respective cluster at which the analysis was performed.

Because amygdala activity has been reported to vary between genders (Schneider et al., 2000; Killgore and Yurgelun-Todd, 2001; Killgore et al., 2001; Thomas et al., 2001), we performed an additional analysis with gender (male, female) as a third between-subject factor. This analysis was regarded to be exploratory because sample sizes of male and female subjects were small (7-8) within each pharmacological group.

\section{Results}

\section{Group characteristics}

The characteristics, physiological measures, and questionnaire results of each pharmacological group are listed in Table 1. Univariate ANOVAs did not reveal any significant between-group differences in age, years of education, IQ, or mood as measured by MWT-B and BDI respectively (all $p$ values $>0.05$ ). Repeatedmeasures ANOVAs (with Greenhouse-Geisser correction for nonsphericity) with the between-subject factor pharmacological group and the within-subject factor measurement time demonstrated that there were no significant main effects or interactions in heart rate, systolic or diastolic blood pressure, or physical complaints ratings (B-L) (all $p$ values $>0.05$ ). In contrast, mood ratings (Bf-S) were influenced by the measurement time $(n=61$, $\left.F_{(1.7,98.0)}=7.40, p<0.002\right)$, which was due to significantly higher ratings indicating impaired mood for measurement 3 (after scanning) when compared with measurement 1 (before drug administration) $\left(t_{(60)}=-3.17, p<0.01\right)$ and measurement 2 (before scanning $)\left(t_{(60)}=-3.00, p<0.01\right)$, as revealed by post hoc paired $t$ tests after correction for multiple testing.

We found no main effect of pharmacological group on Bf-S assessment and no interaction of pharmacological group and measurement time ( $p$ values $>0.05$ ). Thus, subjects tended to 


\section{Table 2. fMRI activations}

\begin{tabular}{|c|c|c|c|c|c|c|c|}
\hline & & $x$ & $y$ & $z$ & Effects & Z & $p$ \\
\hline \multirow{3}{*}{ Basolateral amygdala } & $\mathrm{L}$ & -20 & 2 & -25 & Main effect of emotion & 4.88 & $<0.0005^{*}$ \\
\hline & & & & & $\mathrm{N}>\mathrm{H}$ & 5.17 & $<0.000005$ \\
\hline & & & & & $\mathrm{F}>\mathrm{H}$ & 3.53 & $<0.005$ \\
\hline \multirow[t]{3}{*}{ Basolateral amygdala } & $\mathrm{R}$ & 30 & -2 & -21 & Main effect of emotion & 3.79 & $<0.05^{*}$ \\
\hline & & & & & $\mathrm{N}>\mathrm{H}$ & 4.15 & $<0.00005$ \\
\hline & & & & & $\mathrm{F}>\mathrm{H}$ & 2.53 & $<0.01$ \\
\hline \multirow[t]{3}{*}{ Centromedial amygdala } & $\mathrm{R}$ & 32 & -4 & -11 & Interaction $\mathrm{RBX} \times$ emotion & 3.84 & $<0.05^{*}$ \\
\hline & & & & & $\operatorname{RBX}(F>H)>\operatorname{no} R B X(F>H)$ & 3.90 & $<0.00005$ \\
\hline & & & & & $\operatorname{RBX}(\mathrm{F}>\mathrm{N})>\operatorname{no} \operatorname{RBX}(\mathrm{F}>\mathrm{N})$ & 3.35 & $<0.0005$ \\
\hline \multicolumn{8}{|c|}{ Analysis focused on groups with cortisol administration (Cort-only, Rbx-Cort): } \\
\hline & & & & & Interaction RBX $\times$ emotion & 4.25 & $<0.00005$ \\
\hline & & & & & $\operatorname{RBX}(F>H)>n_{0} R B X(F>H)$ & 4.15 & $<0.00005$ \\
\hline & & & & & $\operatorname{RBX}(\mathrm{F}>\mathrm{N})>\operatorname{no} \operatorname{RBX}(\mathrm{F}>\mathrm{N})$ & 3.86 & $<0.0001$ \\
\hline
\end{tabular}

Brain activations as detected by a repeated-measures ANOVA with the between-subject factors RBX, CORT, and the within-subject factor emotion at $p<0.05$ FWE-corrected. Listed are the effects that yielded significant results and post hoc tests in the respective clusters. An asterisk indicates the significance leve adjusted for the amygdaloid ROIs. For the post hoc tests, the significance levels were adjusted for the size of the respective cluster in which these tests were performed. H, Happy; N, neutral; F, fearful; L, left; R, right.

report a decrease in mood after scanning, regardless of drug intake.

\section{Reaction times during scanning}

A repeated measures ANOVA on reaction times during fMRI scanning (Greenhouse-Geisser corrected for nonsphericity) revealed a main effect of emotion $\left(F_{(1.8,102.8)}=33.54, p<0.0001\right)$. Paired $t$ tests (with Bonferroni correction for multiple comparisons) comprising all pharmacological groups showed that this effect was due to higher reaction times for neutral facial expressions than for happy $\left(t_{(60)}=5.95, p<0.0001\right)$ or fearful facial expressions $\left(t_{(60)}=7.10, p<0.0001\right)$. Reaction times for happy facial expressions did not differ from those for fearful expressions $(p>0.05)$. There were no main effects of NE or CORT; no two-way interactions between NE and emotion, CORT and emotion, NE and CORT; and no three-way interaction between NE, CORT and emotion.

\section{fMRI data: main effect of emotion}

The ROI analysis of the amygdalae revealed a main effect of emotion in the laterobasal nuclei bilaterally (Table 2, Fig. 1a). Subsequent paired $t$ tests revealed that within these clusters, activity was significantly greater for neutral and fearful faces than for happy faces (Table 2, Fig. 1a). The activity for fearful and neutral faces did not significantly differ.

\section{fMRI data: interaction of RBX and emotion}

An interaction effect between RBX and emotion was evident at the border of the right superficial and centromedial nuclei (Table 2, Fig. $1 b$ ). Subsequent tests showed that this was due to greater responses to fearful than to happy or neutral faces in the Rbx-only and Rbx-Cort groups. The inverse pattern was observed in the groups without RBX administration (Plac and Cort-only). (Table 2, Fig. 1b). Next, we tested for the interaction effect of RBX and emotion separately within the CORT groups (Cort-only and Rbx-Cort) and the non-CORT groups (Plac and Rbx-only) because we hypothesized that the influence of CORT on amygdala responses depends on the presence or absence of pharmacologically elevated NE signaling. This analysis showed that the interaction effect of RBX and emotion was mainly driven by opposite patterns of activity in the Cort-only and Rbx-Cort groups (Table 2, Fig. 1b). Subsequent tests demonstrated interaction effects of $\mathrm{RBX} \times$ fearful versus happy and $\mathrm{RBX} \times$ fearful versus neutral (Table 2): while happy faces elicited the highest activity and fear- ful faces elicited the lowest activity in the Cort-only group, fearful faces elicited the highest activity followed by happy and neutral faces in the Rbx-Cort group (Fig. $1 b)$.There was no significant interaction effect of RBX with emotion in the nonCORT groups (Plac and Rbx-only).

Thus, noradrenergic stimulation significantly altered amygdaloid activation patterns in response to stimuli of different emotions under elevated CORT levels. The remaining effects (main effect of RBX, main effect of CORT, interaction between CORT and emotion, interaction between RBX and CORT, and interaction between CORT, RBX and emotion) were not significant (all $p$ values $>0.05$ ).

\section{fMRI data: effects of gender}

There was no main effect nor any interaction effect of gender with other factors (all $p$ values $>0.05$ ).

\section{Postscan arousal ratings}

A repeated measures ANOVA on arousal ratings (GreenhouseGeisser corrected for nonsphericity) revealed a main effect of emotion $\left(F_{(1.7,89.9)}=201.49, p<0.0001\right)$, an interaction of CORT and emotion $\left(F_{(1.66,89.85)}=3.78, p<0.05\right)$, and an interaction of CORT, RBX, and emotion $\left(F_{(1.7,89.9)}=3.73, p<0.05\right)$ (Fig. 2a). To investigate more closely whether CORT interacted differently with arousal ratings in the presence or absence of RBX (based on the three-way interaction), two repeated measures ANOVAs with the factors CORT and emotion were calculated separately for the groups with RBX administration (Rbx-only and Rbx-Cort) and without RBX administration (Plac and Cortonly). In the non-RBX groups, there was an interaction of emotion and $\operatorname{CORT}\left(F_{(1.8,52.6)}=6.90, p<0.005\right)$ and a main effect of emotion $\left.\left(F_{(1.8,52.6)}=92.78\right), p<0.0001\right)$.

Post hoc two-sample $t$ tests revealed that arousal ratings for happy faces were significantly lower in the Cort-only group than in the Plac group $\left(t_{(29)}=2.860, p<0.05\right)$, while there were no differential ratings for neutral or fearful faces. We found no significant interaction of emotion and CORT $(p>0.05)$ but a main effect of emotion $\left.\left(F_{(1.4,34.1)}=117.36\right), p<0.0001\right)$ in the RBX groups. The highest scores were obtained fearful faces followed by happy faces; neutral faces yielded the lowest scores (Fig. 2a).

Within-group paired $t$ tests (Bonferroni-corrected for multiple comparisons) showed that independent of the specific type of drug challenge, fearful and happy faces yielded similar arousal scores $(p>0.05)$ but were rated as more arousing than neutral faces $(p<0.05)$ - with one exception: in the Cort-only group, happy faces were assessed as less arousing than fearful faces $(p<$ $0.05)$ and did not yield significantly different arousal scores than neutral faces $(p>0.05)$ (Fig. $2 a)$.

\section{Postscan valence ratings}

A repeated measures ANOVA on valence rating revealed a main effect of emotion $\left(F_{(1.9,85.4)}=408.83, p<0.0001\right)$, a main effect of CORT $\left(F_{(1,54)}=7.80, p<0.01\right)$, and an interaction of emotion and CORT $\left(F_{(1.9,85.4)}=4.27, p<0.05\right)($ Fig. $2 a)$.

Post hoc two-sample $t$ tests revealed that neutral faces were assessed as more pleasant by the CORT groups (Cort-only and Rbx-Cort) than by the non-CORT groups (Plac and Rbx-only) $\left(t_{(56)}=-3.58, p<0.001\right)$. In contrast, CORT had no influence 
on the assessment of emotional stimulus valence. Within-group paired $t$ tests (Bonferroni-corrected for multiple comparisons) showed that happy faces yielded the highest pleasance scores in all groups, whereas fearful faces yielded the lowest pleasance scores (all $p$ values $<0.001$ ) (Fig. $2 a)$.

\section{Drug levels}

Mean plasma RBX levels and salivary CORT levels are listed in Table 3. A twosample $t$ test revealed no difference in RBX levels between the Rbx-only and the RbxCort group $(p>0.05)$. A sufficient amount of saliva in all three measurements was obtained from 55 of 62 subjects. A repeated measures ANOVA on CORT levels (Greenhouse-Geisser corrected for nonsphericity) with the between-subject factors RBX (RBX, no RBX) and CORT (CORT, no CORT), and with the withinsubject factor measurement time (predrug, prescan, postscan) revealed a main effect of measurement time $\left(F_{(1.1 .57 .7)}=\right.$ 34.06, $p<0.0001)$, a main effect of CORT $\left(F_{(1,51)}=54.26, p<0.0001\right)$, and an interaction of CORT and measurement time $\left(F_{(1.1,57.7)}=38.66, p<0.0001\right)$. Post hoc two-sample $t$ tests did not reveal any significant between-group differences in predrug CORT levels (all $p$ values $>0.05$ ) (Fig. $2 b$ ). As expected, the salivary CORT levels of the Cort-only group and the Rbx-Cort group significantly differed from those of the Plac and Rbx-only group at prescan and postscan measurements (all $p$ values $<0.05$ ) (Fig. 2b). CORT levels differed significantly neither at the prescan nor at the postscan measurements between the Cortonly and the Rbx-Cort group (all $p$ values $>0.05)$. Consistent with the salivary data, plasma total CORT levels were highly correlated with the salivary free CORT levels at measurement $3(n=55, r=0.9453, p<$ 0.00001 ) (Fig. 2b).

\section{Discussion}

In the present study, we manipulated amygdala responses to social-emotional stimuli in healthy volunteers by pharmacologically enhancing the action of two neuromodulators that are endogenously released during acute stress. Specifically, we induced a negativity bias by experimental increase of the synaptic levels of the neurotransmitter NE, evident in a relative decrease in amygdala responses to positive stimuli and a relative increase in amygdala responses to negative stimuli. Interestingly, this neural response bias was most pronounced when NE and CORT signaling were simultaneously challenged.

\section{fMRI data: negativity bias}

Central to our study is the observation that stimulation of NE signaling induced a negativity bias by decreasing amygdala activation to positive stimuli but increasing amygdala activation to negative stim- uli. This pharmacological modulation of amygdaloid responses to social-emotional stimuli we suggest provides a model of how amygdala responsiveness is altered in stressful situations such as anxious states, which are typically accompanied by an increased endogenous release of NE and CORT.

One consistent finding across human fMRI studies is the exquisite sensitivity of the amygdala to social-emotional information provided by faces (Adolphs and Spezio, 2006). Previous literature suggests that the amygdala primarily responds to aversive faces (particularly fearful) (Anderson and Phelps, 2001; Phelps et al., 2001; Reinders et al., 2006) but more recent studies, which often used movies to stimulate the amygdala, indicate that happy faces evoke equal amygdaloid responses (LaBar et al., 2003; Fitzgerald et al., 2006; van der Gaag et al., 2007; Hurlemann et al., 2008; Sergerie et al., 2008), suggesting a lack of emotional selectivity under more ecological conditions. A stress-induced nega- 
a

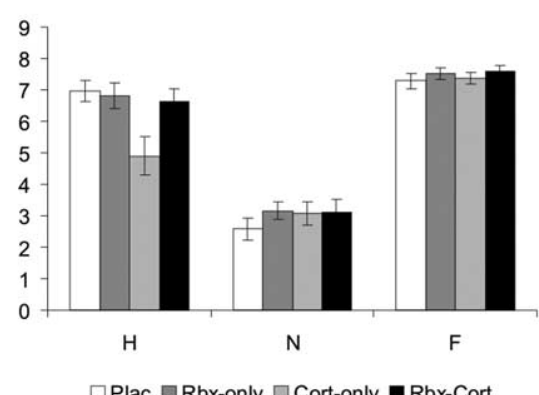

b

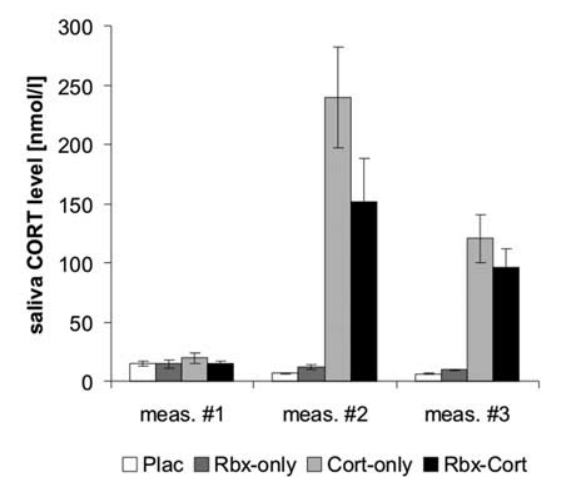

valence
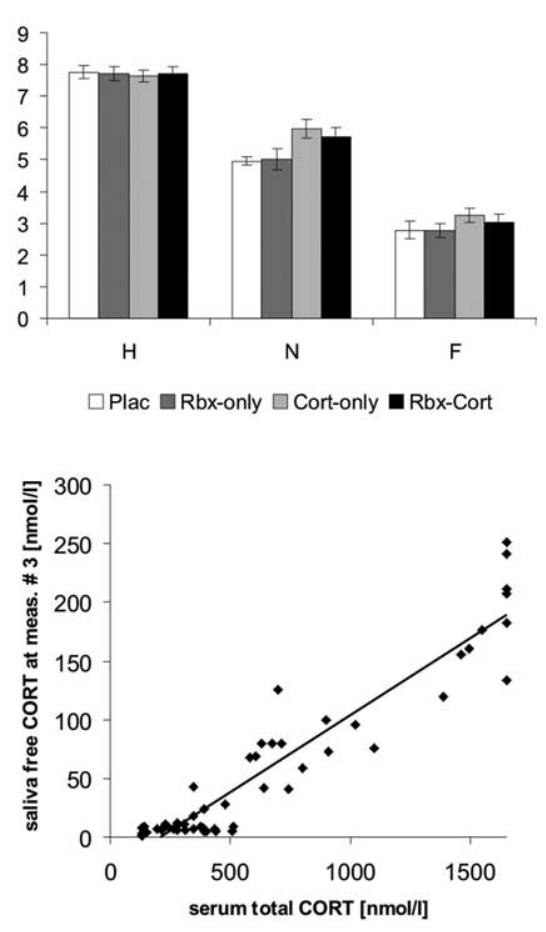

Figure 2. $\quad \boldsymbol{a}$, Rating scores for emotional arousal and pleasure (valence) for the three emotional film categories plotted for each pharmacological group. $\boldsymbol{b}$, Left, Salivary CORT levels at measurements before drug administration, before scanning and after scanning plotted for each pharmacological group. Right, Correlation between salivary free CORT levels after scanning and serum total CORT.

Table 3. Drug levels

\begin{tabular}{lcccc}
\hline & Plac & Cort-only & Rbx-only & Rbx-Cort \\
\hline Saliv. CORT 1 [nmol/l] & $14.5(7.2)$ & $19.6(16.5)$ & $14.7(12.8)$ & $14.7(6.7)$ \\
Saliv. CORT 2 [nmol/l] & $6.4(3.7)$ & $239.6(157.8)$ & $11.6(6.7)$ & $151.1(134.0)$ \\
Saliv. CORT 3 [nmol/l] & $6.1(2.6)$ & $120.4(76.6)$ & $9.4(3.3)$ & $95.6(58.7)$ \\
Serum RBX [ng/ml] & & & $107.5(31.1)$ & $113.8(27.7)$ \\
\hline
\end{tabular}

Salivary CORT levels (1, before drug administration; 2, before scanning; 3, after scanning) and serum RBX levels (after scanning). Saliv., Salivary.

clei of the amygdala, contrary to most structures of the HPA axis, act as a feedforward link by increasing $\mathrm{CRH}$ and ACTH release at elevated glucocorticoid levels (Dallman et al., 2003; Herman et al., 2005). We speculate that under pathological conditions, the amygdala may override the protective feedback loops of the HPA axis, thus effectively contributing to its own hyperreagibility.

At first glance, the present results are in contrast with the study by Norbury et al. (2007) who reported decreased amygdala responses to covert fearful faces after subchronic RBX treatment over $7 \mathrm{~d}$. This discrepancy is most likely due to methodological differences: In our study, only a single clinical dose of RBX was administered. Furthermore, the interaction between RBX and emotion observed in our study was mainly mediated by NE-CORT interactions following co-administration of RBX and CORT rather than RBX alone. These effects are unlikely to be caused by a pharmacological modulation of the BOLD signal per se since there were no significant main effects of RBX or CORT.

\section{Glucocorticoid-noradrenergic interplay in the amygdala}

Our data demonstrate that elevated NE signaling modulates neural activity in the human amygdala particularly in the presence of elevated CORT signaling, suggesting an interdependence of NE and CORT in their neuronal action. The presence of $\mathrm{NE}$ is critical for the amygdala-mediated enhancement of memory for socialemotional stimuli in rodents (Quirarte et al., 1997; Sheline et al., 2001; McGaugh and Roozendaal, 2002) and humans

tivity bias, however, may amplify the cognitive assessment of and lead to quicker physiological reactions to aversive information at the cost of appetitive information. Crucially, such preferential processing of aversive information is typically observed in MDD patients (Beck, 1967; Chamberlain and Sahakian, 2006). The present study demonstrates that even in young healthy individuals, an elevation of NE and CORT signaling alters amygdaloid activity. Given this empirical background, we speculate that a tonic upregulation of the central noradrenergic system in combination with a dysregulated HPA axis may induce a pathological hyperreagibility of the amygdala to emotionally negative stimuli (Phan et al., 2006; Dannlowski et al., 2007; Goossens et al., 2007; Stein et al., 2007). The present findings may thus shed new light on how stress and thereby elevated stress hormone levels could alter amygdaloid function in a way that eventually predisposes an individual to AXD or MDD (Young et al., 2004; Burke et al., 2005; Southwick et al., 2005). In support of this view, individuals with increased sympathetic responses have been identified to be particularly vulnerable to developing these pathologies (Southwick et al., 2005).

On a functional level, amygdaloid hyperactivity may even reinforce the dysregulation of the HPA axis: the centromedial nu-
(Strange et al., 2003; Strange and Dolan, 2004; van Stegeren et al., 2005).

Elevations of CORT activity in the brain exert modulatory effects on amygdala function during encoding (Cahill et al., 2003; Hurlemann et al., 2007) and consolidation (Buchanan and Lovallo, 2001; Roozendaal, 2002; Abercrombie et al., 2003) of emotional stimuli. Importantly, however, studies in rodents (Quirarte et al., 1997; Roozendaal et al., 2006b) and humans (van Stegeren et al., 2007) suggest that the memory-modulating effects of CORT, released during stress, require NE co-activation of the amygdala. The present findings bridge the gap to models derived from studies in rodents, which show that noradrenergic activation of the amygdala is a crucial prerequisite for the neural effects of CORT (Roozendaal et al., 2006a,b). On a behavioral level, a blockade of $\beta$-adrenergic receptors abolishes the negative effect of CORT on memory retrieval in humans (de Quervain et al., 2007). While there is indirect evidence from fMRI studies for NE-CORT interactions in increasing amygdala responses to emotional stimuli (van Stegeren et al., 2008), the present study is the first to directly demonstrate such changes by pharmacological manipulation of NE and CORT signaling. Some caution is advised regarding the interpretation that the observed effects reflect 
direct NE-CORT interactions in the amygdala (Roozendaal et al., 2006a,b). Based on the present analysis, we cannot rule out that some of the activation differences are mediated by remote effects from other brain areas.

The interaction effect between NE and emotion found in our study differs in anatomical localization from where it would have been expected based on studies in rodents. While these studies indicate that the basolateral but not the centromedial nuclei of the amygdala are susceptible to glucocorticoid action (Roozendaal and McGaugh, 1996, 1997; Roozendaal et al., 2006b), our findings reveal that in humans the centromedial and superficial amygdalar nuclei are most sensitive to the modulatory effects of NE and CORT. Such divergent anatomical localization might result from technical limitations regarding image acquisition and/or preprocessing, thus complicating the probabilistic assignment of activation sites to amygdalar nuclei. However, given the consistencies with our previous work (Hurlemann et al., 2008), we suggest that the observed discrepancy most likely reflects a different functional specialization of homologous nuclei between species, limiting the extrapolation of functional and pharmacological properties of these nuclei in rodents to the human amygdala.

\section{Amygdala response to facial emotion}

As expected from previous studies we observed a differential bilateral activation of the laterobasal amygdala in response to dynamic facial expressions, regardless of the specific pharmacological challenge. Fearful faces elicited greater activity than happy faces in this subregion, which is in line with the majority of electrophysiological and fMRI studies in rodents and primates (Gothard et al., 2007; Hoffman et al., 2007; Rolls, 2007). The highest activity, however, was observed in response to neutral facial expressions. While this result conflicts with initial reports of the greatest amygdala responses being to static fearful (Vuilleumier et al., 2001; Canli et al., 2002) or happy faces (Breiter et al., 1996; Pessoa et al., 2002), it corroborates findings from more recent investigations, partly derived from dynamic face stimuli, which document robust amygdala activations to neutral faces (LaBar et al., 2003; Fitzgerald et al., 2006; van der Gaag et al., 2007; Sergerie et al., 2008). In our study, the strongest amygdala response to emotionally neutral movies (female and male actors blowing up their cheeks) might have been evoked by the, at least initially, ambiguous nature of these stimuli (see also Zald, 2003). This ambiguity is also reflected in largest push-button response latencies for this specific stimulus type in the emotion discrimination task. Interestingly, the present findings slightly differ from those reported by van der Gaag et al. (2007), who did not detect any differences between amygdala responses to neutral and emotional film clips. Advances in study design and methodology (including a larger number of subjects) might account for higher statistical power and improved response segregation in the present study.

The observation that the amygdala activity pattern paralleled neither the postscan arousal nor valence ratings may be due to the fact that the amygdala is the first in a line of interconnected neural modules that precategorizes incoming sensory stimuli with respect to their social-emotional significance (Dolan, 2002; Ohman et al., 2007). This view does not conflict with increasing evidence that amygdaloid activity is under modulatory top-down control by higher-order cortical areas (Ochsner and Gross, 2005; Cunningham et al., 2008) and is susceptible to fluctuations in attentional resources devoted to the processing of social-emotional information (Bishop et al., 2007;Silvert et al., 2007; Pessoa, 2008).
Although some studies reported a close match between ratings and amygdala activity (Hariri et al., 2000; Lieberman et al., 2007), we suggest that the postscan assessments are most likely reflective of higher-order cognitive judgments orchestrated by prefrontal subregions (Dolcos et al., 2004). The postscan ratings primarily served to validate an emotional salience that the stimuli were supposed to convey, and revealed a similar pattern for each pharmacological group. The observed tendency of CORT to reduce appetitive stimulus-evoked arousal might reflect the distorting influence of hypercortisolism on higher-order cognitive appraisal processes in AXD and MDD (Young et al., 2004; Burke et al., 2005) and definitely merits further investigation.

\section{Conclusions}

By means of pharmacological enhancement of NE and CORT signaling, we induced a negative response bias in the human amygdala that did not exist at placebo baseline. This finding in healthy humans replicates data obtained in rodents and implicates a causative role of NE-CORT interactions in the emergence of a negative bias of cognitive and emotional functions, which is germane in stress-related affective spectrum disorders.

\section{References}

Abelson JL, Curtis GC (1996) Hypothalamic-pituitary-adrenal axis activity in panic disorder. 24-hour secretion of corticotropin and cortisol. Arch Gen Psychiatry 53:323-331.

Abercrombie HC, Kalin NH, Thurow ME, Rosenkranz MA, Davidson RJ (2003) Cortisol variation in humans affects memory for emotionally laden and neutral information. Behav Neurosci 117:505-516.

Adolphs R, Spezio M (2006) Role of the amygdala in processing visual social stimuli. Prog Brain Res 156:363-378.

Amunts K, Kedo O, Kindler M, Pieperhoff P, Mohlberg H, Shah NJ, Habel U, Schneider F, Zilles K (2005) Cytoarchitectonic mapping of the human amygdala, hippocampal region and entorhinal cortex: intersubject variability and probability maps. Anat Embryol (Berl) 210:343-352.

Anderson AK, Phelps EA (2001) Lesions of the human amygdala impair enhanced perception of emotionally salient events. Nature 411:305-309.

Asan E (1998) The catecholaminergic innervation of the rat amygdala. Adv Anat Embryol Cell Biol 142:1-118.

Beck AT (1967) Depression: clinical, experimental, and theoretical aspects. New York: Hoeber.

Beck AT, Hautzinger M, Bailer M, Worall H, Keller F (1995) BeckDepressions-Inventar (BDI) 2, überarbeitete Auflage. Göttingen: Hogrefe.

Berridge CW, Waterhouse BD (2003) The locus coeruleus-noradrenergic system: modulation of behavioral state and state-dependent cognitive processes. Brain Res Brain Res Rev 42:33-84.

Bishop SJ, Jenkins R, Lawrence AD (2007) Neural processing of fearful faces: effects of anxiety are gated by perceptual capacity limitations. Cereb Cortex 17:1595-1603.

Breiter HC, Etcoff NL, Whalen PJ, Kennedy WA, Rauch SL, Buckner RL, Strauss MM, Hyman SE, Rosen BR (1996) Response and habituation of the human amygdala during visual processing of facial expression. Neuron 17:875-887.

Buchanan TW, Lovallo WR (2001) Enhanced memory for emotional material following stress-level cortisol treatment in humans. Psychoneuroendocrinology 26:307-317.

Burke HM, Davis MC, Otte C, Mohr DC (2005) Depression and cortisol responses to psychological stress: a meta-analysis. Psychoneuroendocrinology 30:846-856.

Cahill L, Gorski L, Le K (2003) Enhanced human memory consolidation with post-learning stress: interaction with the degree of arousal at encoding. Learn Mem 10:270-274.

Canli T, Sivers H, Whitfield SL, Gotlib IH, Gabrieli JD (2002) Amygdala response to happy faces as a function of extraversion. Science 296:2191.

Chamberlain SR, Sahakian BJ (2006) The neuropsychology of mood disorders. Curr Psychiatry Rep 8:458-463.

Charney DS (2004) Psychobiological mechanisms of resilience and vulnerability: implications for successful adaptation to extreme stress. Am J Psychiatry 161:195-216. 
Cunningham WA, Van Bavel JJ, Johnsen IR (2008) Affective flexibility: evaluative processing goals shape amygdala activity. Psychol Sci 19:152-160.

Czock D, Keller F, Rasche FM, Häussler U (2005) Pharmacokinetics and pharmacodynamics of systemically administered glucocorticoids. Clin Pharmacokinet 44:61-98.

Dallman MF, Pecoraro N, Akana SF, La Fleur SE, Gomez F, Houshyar H, Bell ME, Bhatnagar S, Laugero KD, Manalo S (2003) Chronic stress and obesity: a new view of "comfort food". Proc Natl Acad Sci U S A 100:11696-11701.

Dannlowski U, Ohrmann P, Bauer J, Kugel H, Arolt V, Heindel W, Kersting A, Baune BT, Suslow T (2007) Amygdala reactivity to masked negative faces is associated with automatic judgmental bias in major depression: a 3 T fMRI study. J Psychiatry Neurosci 32:423-429.

Deichmann R, Gottfried JA, Hutton C, Turner R (2003) Optimized EPI for fMRI studies of the orbitofrontal cortex. Neuroimage 19:430-441.

de Kloet ER (2003) Hormones, brain and stress. Endocr Regul 37:51-68.

de Quervain DJ, Aerni A, Roozendaal B (2007) Preventive effect of betaadrenoceptor blockade on glucocorticoid-induced memory retrieval deficits. Am J Psychiatry 164:967-969.

Dolan RJ (2002) Emotion, cognition, and behavior. Science 298:1191-1194.

Dolcos F, LaBar KS, Cabeza R (2004) Dissociable effects of arousal and valence on prefrontal activity indexing emotional evaluation and subsequent memory: an event-related fMRI study. Neuroimage 23:64-74.

Eickhoff SB, Stephan KE, Mohlberg H, Grefkes C, Fink GR, Amunts K, Zilles K (2005) A new SPM toolbox for combining probabilistic cytoarchitectonic maps and functional imaging data. Neuroimage 25:1325-1335.

Eickhoff SB, Heim S, Zilles K, Amunts K (2006) Testing anatomically specified hypotheses in functional imaging using cytoarchitectonic maps. Neuroimage 32:570-582.

Etkin A, Wager TD (2007) Functional neuroimaging of anxiety: a metaanalysis of emotional processing in PTSD, social anxiety disorder, and specific phobia. Am J Psychiatry 164:1476-1488.

Fitzgerald DA, Angstadt M, Jelsone LM, Nathan PJ, Phan KL (2006) Beyond threat: amygdala reactivity across multiple expressions of facial affect. Neuroimage 30:1441-1448.

Fleishaker JC (2000) Clinical pharmacokinetics of reboxetine, a selective norepinephrine reuptake inhibitor for the treatment of patients with depression. Clin Pharmacokinet 39:413-427.

Gold PW, Goodwin FK, Chrousos GP (1988) Clinical and biochemical manifestations of depression. Relation to the neurobiology of stress (1). N Engl J Med 319:348-353.

Goossens L, Sunaert S, Peeters R, Griez EJ, Schruers KR (2007) Amygdala hyperfunction in phobic fear normalizes after exposure. Biol Psychiatry 62:1119-1125.

Gothard KM, Battaglia FP, Erickson CA, Spitler KM, Amaral DG (2007) Neural responses to facial expression and face identity in the monkey amygdala. J Neurophysiol 97:1671-1683.

Hariri AR, Bookheimer SY, Mazziotta JC (2000) Modulating emotional responses: effects of a neocortical network on the limbic system. Neuroreport 11:43-48.

Herman JP, Ostrander MM, Mueller NK, Figueiredo H (2005) Limbic system mechanisms of stress regulation: hypothalamo-pituitaryadrenocortical axis. Prog Neuropsychopharmacol Biol Psychiatry 29:1201-1213.

Hoffman KL, Gothard KM, Schmid MC, Logothetis NK (2007) Facialexpression and gaze-selective responses in the monkey amygdala. Curr Biol 17:766-772.

Hurlemann R, Hawellek B, Matusch A, Kolsch H, Wollersen H, Madea B, Vogeley K, Maier W, Dolan RJ (2005) Noradrenergic modulation of emotion-induced forgetting and remembering. J Neurosci 25:6343-6349.

Hurlemann R, Matusch A, Hawellek B, Klingmuller D, Kolsch H, Maier W, Dolan RJ (2007) Emotion-induced retrograde amnesia varies as a function of noradrenergic-glucocorticoid activity. Psychopharmacology (Berl) 194:261-269.

Hurlemann R, Rehme AK, Diessel M, Kukolja J, Maier W, Walter H, Cohen MX (2008) Segregating intra-amygdalar responses to dynamic facial emotion with cytoarchitectonic maximum probability maps. J Neurosci Methods 172:13-20.

Joëls M (2006) Corticosteroid effects in the brain: U-shape it. Trends Pharmacol Sci 27:244-250.
Kent JM (2000) SNaRIs, NaSSAs, and NaRIs: new agents for the treatment of depression. Lancet 355:911-918.

Kiebel S, Holmes AP (2003) The General Linear Model. In: Human Brain Function (Frackowiak RSJ, Friston K, Frith CD, Dolan RJ, Price CJ, Ashburner J, Penny WD, eds), pp 725-760. San Diego: Academic.

Killgore WD, Yurgelun-Todd DA (2001) Sex differences in amygdala activation during the perception of facial affect. Neuroreport 12:2543-2547.

Killgore WD, Oki M, Yurgelun-Todd DA (2001) Sex-specific developmental changes in amygdala responses to affective faces. Neuroreport 12:427-433.

Kuraoka K, Nakamura K (2007) Responses of single neurons in monkey amygdala to facial and vocal emotions. J Neurophysiol 97:1379-1387.

LaBar KS, Crupain MJ, Voyvodic JT, McCarthy G (2003) Dynamic perception of facial affect and identity in the human brain. Cereb Cortex 13:1023-1033.

Lecrubier Y, Sheehan D, Weiler E, Amorim P, Bonora I, Sheehan K, Janavs J, Dunbar G (1997) The MINI International Neuropsychiatric Interview (M.I.N.I.) A Short Diagnostic Structured Interview: Reliability and Validity According to the CIDI. Eur Psychiatry 12:224-231.

Lehrl S (1995) Mehrfachwahl-Wortschatz-Test (MWT-B). Erlangen: Straube.

Lieberman MD, Eisenberger NI, Crockett MJ, Tom SM, Pfeifer JH, Way BM (2007) Putting feelings into words: affect labeling disrupts amygdala activity in response to affective stimuli. Psychol Sci 18:421-428.

McGaugh JL, Roozendaal B (2002) Role of adrenal stress hormones in forming lasting memories in the brain. Curr Opin Neurobiol 12:205-210.

Norbury R, Mackay CE, Cowen PJ, Goodwin GM, Harmer CJ (2007) Shortterm antidepressant treatment and facial processing. Functional magnetic resonance imaging study. Br J Psychiatry 190:531-532.

Norbury R, Mackay CE, Cowen PJ, Goodwin GM, Harmer CJ (2008) The effects of reboxetine on emotional processing in healthy volunteers: an fMRI study. Mol Psychiatry 13:1011-1020.

Ochsner KN, Gross JJ (2005) The cognitive control of emotion. Trends Cogn Sci 9:242-249.

Ohman A, Carlsson K, Lundqvist D, Ingvar M (2007) On the unconscious subcortical origin of human fear. Physiol Behav 92:180-185.

Oldfield RC (1971) The assessment and analysis of handedness: the Edinburgh inventory. Neuropsychologia 9:97-113.

Papps BP, Shajahan PM, Ebmeier KP, O'Carroll RE (2002) The effects of noradrenergic re-uptake inhibition on memory encoding in man. Psychopharmacology (Berl) 159:311-318.

Penny WD, Holmes AP (2003) Random effects analysis. In: Human Brain Function (Frackowiak RSJ, Friston KJ, Frith CD, Dolan RJ, Price CJ, Ashburner J, Penny WD, eds), pp 843-850. San Diego: Academic.

Pessoa L (2008) On the relationship between emotion and cognition. Nat Rev Neurosci 9:148-158

Pessoa L, McKenna M, Gutierrez E, Ungerleider LG (2002) Neural processing of emotional faces requires attention. Proc Natl Acad Sci U S A 99:11458-11463.

Phan KL, Fitzgerald DA, Nathan PJ, Tancer ME (2006) Association between amygdala hyperactivity to harsh faces and severity of social anxiety in generalized social phobia. Biol Psychiatry 59:424-429.

Phelps EA, O'Connor KJ, Gatenby JC, Gore JC, Grillon C, Davis M (2001) Activation of the left amygdala to a cognitive representation of fear. Nat Neurosci 4:437-441.

Quirarte GL, Roozendaal B, McGaugh JL (1997) Glucocorticoid enhancement of memory storage involves noradrenergic activation in the basolateral amygdala. Proc Natl Acad Sci U S A 94:14048-14053.

Reinders AA, Gläscher J, de Jong Jr, Willemsen AT, den Boer JA, Büchel C (2006) Detecting fearful and neutral faces: BOLD latency differences in amygdala-hippocampal junction. Neuroimage 33:805-814.

Rolls ET (2007) The representation of information about faces in the temporal and frontal lobes. Neuropsychologia 45:124-143.

Roozendaal B (2002) Stress and memory: opposing effects of glucocorticoids on memory consolidation and memory retrieval. Neurobiol Learn Mem 78:578-595.

Roozendaal B, McGaugh JL (1996) Amygdaloid nuclei lesions differentially affect glucocorticoid-induced memory enhancement in an inhibitory avoidance task. Neurobiol Learn Mem 65:1-8.

Roozendaal B, McGaugh JL (1997) Glucocorticoid receptor agonist and antagonist administration into the basolateral but not central amygdala modulates memory storage. Neurobiol Learn Mem 67:176-179. 
Roozendaal B, Hui GK, Hui IR, Berlau DJ, McGaugh JL, Weinberger NM (2006a) Basolateral amygdala noradrenergic activity mediates corticosterone-induced enhancement of auditory fear conditioning. Neurobiol Learn Mem 86:249-255.

Roozendaal B, Okuda S, Van der Zee EA, McGaugh JL (2006b) Glucocorticoid enhancement of memory requires arousal-induced noradrenergic activation in the basolateral amygdala. Proc Natl Acad Sci U S A 103:6741-6746.

Scates AC, Doraiswamy PM (2000) Reboxetine: a selective norepinephrine reuptake inhibitor for the treatment of depression. Ann Pharmacother 34:1302-1312.

Schneider F, Habel U, Kessler C, Salloum JB, Posse S (2000) Gender differences in regional cerebral activity during sadness. Hum Brain Mapp 9:226-238.

Sergerie K, Chochol C, Armony JL (2008) The role of the amygdala in emotional processing: a quantitative meta-analysis of functional neuroimaging studies. Neurosci Biobehav Rev 32:811-830.

Sheline YI, Barch DM, Donnelly JM, Ollinger JM, Snyder AZ, Mintun MA (2001) Increased amygdala response to masked emotional faces in depressed subjects resolves with antidepressant treatment: an fMRI study. Biol Psychiatry 50:651-658.

Siegle GJ, Steinhauer SR, Thase ME, Stenger VA, Carter CS (2002) Can't shake that feeling: event-related fMRI assessment of sustained amygdala activity in response to emotional information in depressed individuals. Biol Psychiatry 51:693-707.

Silvert L, Lepsien J, Fragopanagos N, Goolsby B, Kiss M, Taylor JG, Raymond JE, Shapiro KL, Eimer M, Nobre AC (2007) Influence of attentional demands on the processing of emotional facial expressions in the amygdala. Neuroimage 38:357-366.

Southwick SM, Vythilingam M, Charney DS (2005) The psychobiology of depression and resilience to stress: implications for prevention and treatment. Annu Rev Clin Psychol 1:255-291.

Stein MB, Goldin PR, Sareen J, Zorrilla LT, Brown GG (2002) Increased amygdala activation to angry and contemptuous faces in generalized social phobia. Arch Gen Psychiatry 59:1027-1034.

Stein MB, Simmons AN, Feinstein JS, Paulus MP (2007) Increased amygdala and insula activation during emotion processing in anxiety-prone subjects. Am J Psychiatry 164:318-327.

Strange BA, Dolan RJ (2004) Beta-adrenergic modulation of emotional memory-evoked human amygdala and hippocampal responses. Proc Natl Acad Sci U S A 101:11454-11458.

Strange BA, Hurlemann R, Dolan RJ (2003) An emotion-induced retrograde amnesia in humans is amygdala- and beta-adrenergic-dependent. Proc Natl Acad Sci U S A 100:13626-13631.
Thomas KM, Drevets WC, Whalen PJ, Eccard CH, Dahl RE, Ryan ND, Casey BJ (2001) Amygdala response to facial expressions in children and adults. Biol Psychiatry 49:309-316.

van der Gaag C, Minderaa RB, Keysers C (2007) The BOLD signal in the amygdala does not differentiate between dynamic facial expressions. Social, Cognitive and Affective Neuroscience 2:93-103.

van Stegeren AH, Goekoop R, Everaerd W, Scheltens P, Barkhof F, Kuijer JP, Rombouts SA (2005) Noradrenaline mediates amygdala activation in men and women during encoding of emotional material. Neuroimage 24:898-909.

van Stegeren AH, Wolf OT, Everaerd W, Scheltens P, Barkhof F, Rombouts SA (2007) Endogenous cortisol level interacts with noradrenergic activation in the human amygdala. Neurobiol Learn Mem 87:57-66.

van Stegeren AH, Wolf OT, Everaerd W, Rombouts SA (2008) Interaction of endogenous cortisol and noradrenaline in the human amygdala. Prog Brain Res 167:263-268.

van West D, Claes S, Sulon J, Deboutte D (2008) Hypothalamic-pituitaryadrenal reactivity in prepubertal children with social phobia. J Affect Disord 111:281-290.

von Zerssen D, Koeller DM (1976a) Die Befindlichkeitsskala. Weinheim, Germany: Beltz.

von Zerssen D, Koeller DM (1976b) Die Beschwerdeliste. Weinheim, Germany: Beltz.

Vuilleumier P, Armony JL, Driver J, Dolan RJ (2001) Effects of attention and emotion on face processing in the human brain: an event-related fMRI study. Neuron 30:829-841.

Weiskopf N, Hutton C, Josephs O, Deichmann R (2006) Optimal EPI parameters for reduction of susceptibility-induced BOLD sensitivity losses: a whole-brain analysis at $3 \mathrm{~T}$ and $1.5 \mathrm{~T}$. Neuroimage 33:493-504.

Wong ML, Kling MA, Munson PJ, Listwak S, Licinio J, Prolo P, Karp B, McCutcheon IE, Geracioti TD Jr, DeBellis MD, Rice KC, Goldstein DS, Veldhuis JD, Chrousos GP, Oldfield EH, McCann SM, Gold PW (2000) Pronounced and sustained central hypernoradrenergic function in major depression with melancholic features: relation to hypercortisolism and corticotropin-releasing hormone. Proc Natl Acad Sci U S A 97:325-330.

Worsley KJ, Marrett S, Neelin P, Vandal AC, Friston KJ, Evans AC (1996) A unified statistical approach for determining significant signals in images of cerebral activation. Hum Brain Mapp 4:58-74.

Young EA, Abelson JL, Cameron OG (2004) Effect of comorbid anxiety disorders on the hypothalamic-pituitary-adrenal axis response to a social stressor in major depression. Biol Psychiatry 56:113-120.

Zald DH (2003) The human amygdala and the emotional evaluation of sensory stimuli. Brain Res Brain Res Rev 41:88-123. 